\title{
Retroduodenal, retrogastric colon: First case report
}

\author{
Vasanth Stalin ${ }^{1 *}$ and Ravi Teja Pasam ${ }^{2}$ \\ ${ }^{1}$ Ascension St. Mary's Hospital, Saginaw, Michigan, USA \\ ${ }^{2}$ Lahey Clinic, Burlington, Massachusetts, USA
}

\begin{abstract}
We report the first case of retroduodenal, retrogastric colon. We noticed this rare anatomic variant incidentally during a laparoscopic sleeve gastrectomy. Our patient did not have any symptoms secondary to the colonic interposition between stomach, duodenum and pancreas. We had to take down adhesions between the colon and posterior aspect of the stomach to complete the procedure safely, which led to a slightly longer duration of surgery. There were no complications and post-operative course was uneventful. Retrogastric and retroduodenal colons have been reported separately in the past. These anatomic variants are usually asymptomatic and have been documented in the past on CT images and Barium studies. There was only one Case report of symptomatic Retrogastric colon requiring surgical correction of the position. In conclusion, these colonic variants are rare and asymptomatic. Encountering these variants during an unrelated abdominal surgery may necessitate additional steps to perform the procedure safely.
\end{abstract}

\section{Introduction}

Colonic interposition is a rare anatomic variant of the colon where the colon is found interposed between various abdominal organs. The Chilaiditi syndrome is one of the well-documented colonic variants where the hepatic flexure of the colon is interposed between the liver and right hemidiaphragm $[1,2]$. Some of the lesser-known variants are interposition between spleen and diaphragm, retrogastric [3] and retroduodenal 4 colonic interposition. In retrogastric and retroduodenal interposition, the colon is interposed between the pancreas and respective organs. Here, we report a case of retrogastric, retroduodenal colon that was incidentally found intraoperatively.

\section{Case Report}

A 40-year-old Morbidly obese female had presented to our Center for Bariatric Surgery. Her past surgical history was unremarkable. We decided to perform a Laparoscopic Sleeve gastrectomy after the patient went through the rigorous preop process at our Weight loss clinic. During the procedure, it was obvious that the Transverse colon was not present in its usual anatomic position. The colon was noted to be behind the stomach and duodenum (Figure 1). We ran the course of the colon and there was no evidence of additional variants of interposition. Once the greater omentum was transected close to the greater curvature, we identified the colon lying posterior to the stomach and also posterior to the first part of the duodenum. The adhesions between the colon and the posterior aspect of the stomach were taken down in order to fully mobilize the stomach so that the Sleeve gastrectomy could be performed safely. The patient was discharged on post-operative day 1 after tolerating a Stage I Bariatric diet as per our protocol. The patient's longer-term post-operative course was also uneventful over the next year.

\section{Discussion}

This is the first Case report of retrogastric retroduodenal colon. Cases of colonic interposition between the liver and right hemidaphragm and associated symptoms have been well-described in

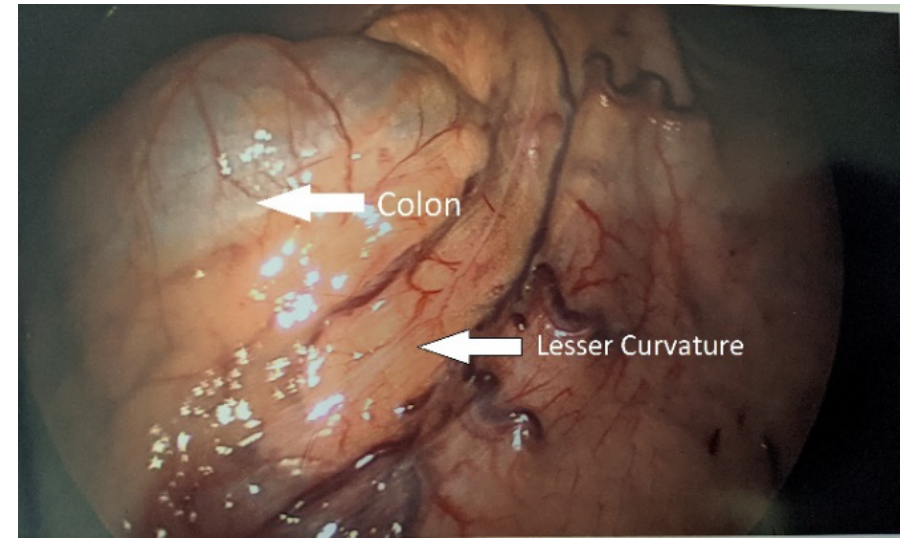

Figure 1. Relation of lesser curvature to the retrogastric colon that has thin overlying pars flaccida

the literature. The less common variants such as retrogastric colon and retroduodneal colon [4] were reported separately in the past but never together in the same patient. These variants have been documented in the past as incidental findings by Radiologists in their analysis of Barium studies and CT images [3-9] and there is only one reported case where patient had to be operated on due to symptoms attributable to the retrogastric colon8.

Retrogastric colon was first reported by Oldfield and Wilbur as an incidental finding in 1993 as a part of their analysis of 9,400 computerized tomography (CT) scans [3]. They reported an incidence

${ }^{\star}$ Correspondence to: Vasanth Stalin, Ascension St. Mary's Hospital, Saginaw, Michigan, USA, E-mail: dr_vasanth@yahoo.com

Key words: retrogastric colon, retroduodenal colon, colonic interposition

Received: December 14, 2020; Accepted: December 23, 2020; Published: December 28, 2020 
of $0.2 \%$, which was similar to the incidence reported by Bredolo, et al. (0.3\%) in their analysis of 4,338 CT scans5 and by Unal, et al. (0.2\%) in 296 CT scans [9]. Oldfield and Wilbur classified the retrogastric colon into type I, type II and type III. Type I was defined as interposition of the colon immediately posterior to the stomach along with incomplete small bowel rotation or malrotation. Type II is the same as Type I but without small bowel rotation abnormalities. Type III was defined as position of splenic flexure of the colon posterior to the spleen without small bowel malrotations. Although they considered Type III as retrogastric colon, we agree with Bredolo, et al's comment that it should not be considered as a retrogastric colon [5]. Bredolo, et al. reported 12 cases in their review of CT scans and found that none of those patients were symptomatic because of the retrogastric colon. On, the other hand we could find only one case report of a retroduodenal colon that was reported by Poppel.

Etiology of the retrogastric colon is unclear. Oldfield and Wilbur postulated that it could be secondary to short mesocolon or embryologic abnormality of bowel fixation based on their interpretation of radiological images [3]. They also noted that it could be just the position of the colon without any above-mentioned abnormalities as there are no direct surgical or autopsy findings to prove this. Harpain, et al. did not mention about the length of the mesocolon in their intraoperative findings8. Our intraoperative findings did not suggest any obvious short mesocolon.

These retrogastric and retroduodenal positions of colon seem to be asymptomatic, which is the case in our patient as well. There is only one case report of symptomatic retrogastric colon published by Harpain, et al [8]. The authors reported that the patient had abdominal pain that was aggravated several hours after meals, most frequently around 4 am in the morning, which was relieved with defecation. The patient was misdiagnosed with Irritable Bowel Syndrome and treated for the same. Lack of symptom resolution led to a more extensive work-up that revealed kinked retrogastric Transverse colon that was interposed between the stomach and pancreas. Laparoscopic lysis of adhesions between the colon, stomach and pancreas was done and the patient did not have recurrence of symptoms post-operatively.

In our patient, the laparoscopic Sleeve gastrectomy took slightly longer than anticipated because of the adhesions between the stomach and transverse colon. We were able to perform the procedure safely without any complications and it did not impact the patient's postoperative course.

In conclusion, retrogastric and retroduodeanal colonic interpositions are rare and asymptomatic. When these variants are found on cross-sectional imaging in patients with abdominal symptoms that do not fit any particular clinical picture, they should be considered as a possible source of symptoms. When found incidentally during abdominal surgeries, these anatomic variants may necessitate lysis of adhesions and do not impact the overall outcome of the procedure (Figure 2-8).

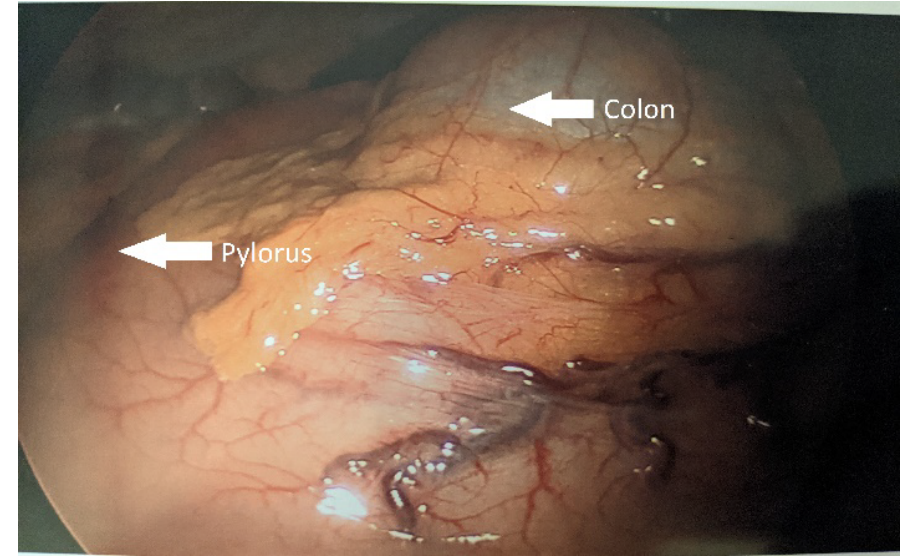

Figure 2. Relation of lesser curvature to the pylorus

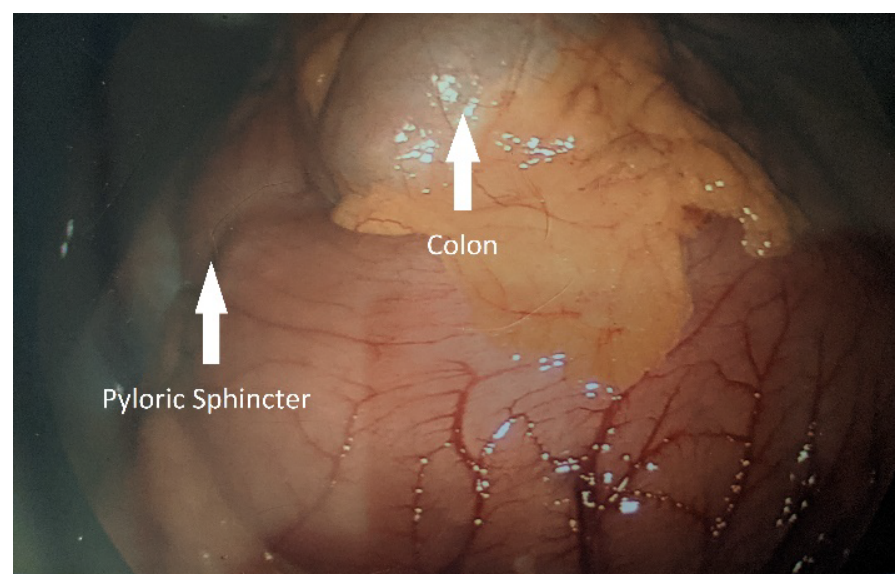

Figure 3. Relation of pyloric to the retrogastric colon

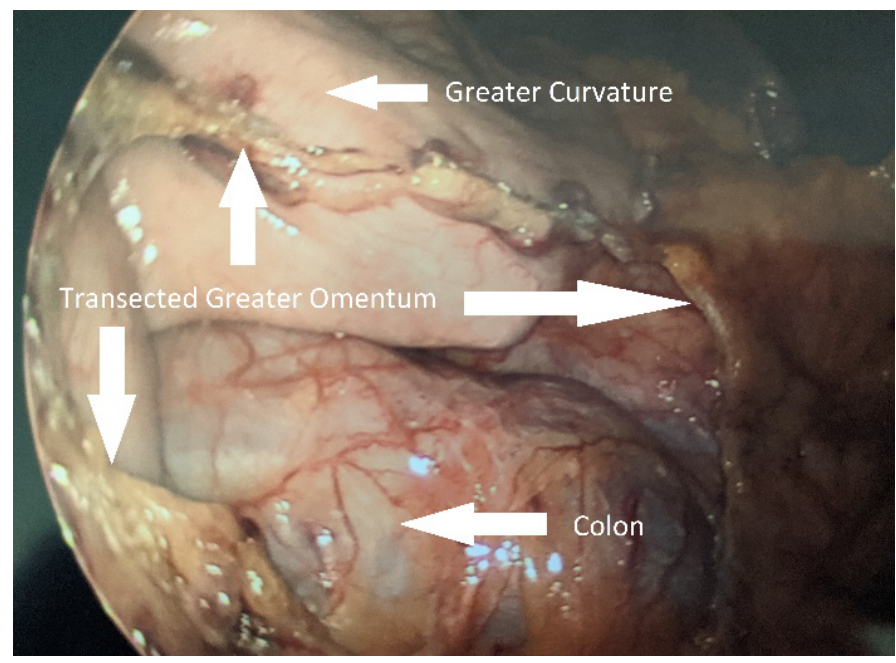

Figure 4. Relation of greater curvature to the retrogastric colon after transection of the greater omentum 


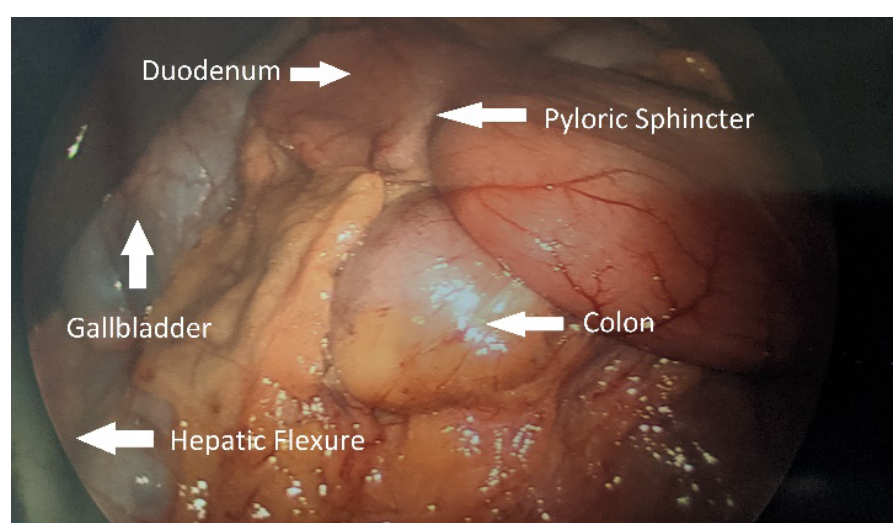

Figure 5. Relation of retrogastric colon to stomach and duodenum after dissection of the distal portion of stomach

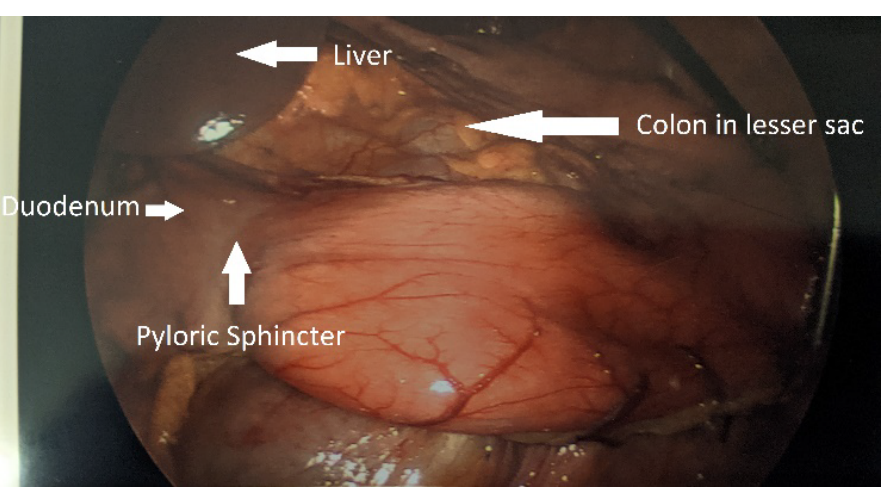

Figure 6. Relation of retrogastric colon in the lesser sac and its relation to stomach, duodenum and liver

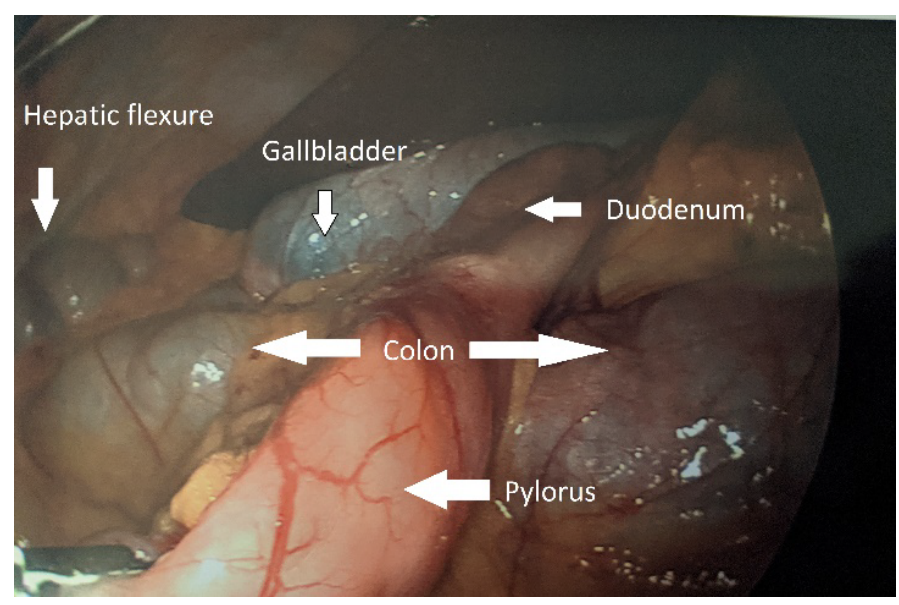

Figure 7. Relation of colon to pylorus, duodenum, liver and gallbladder

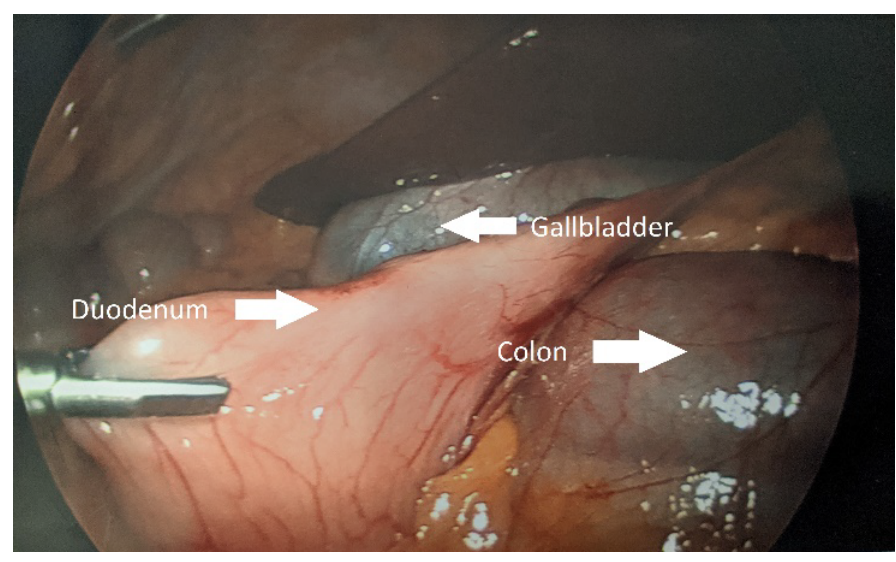

Figure 8. Relation of colon to duodenum, liver and gallbladder

\section{References}

1. Chilaiditi D (1910) Zur Frage der Hepatoptose und Ptose im allgemeinen im Anschluss a drei Falle von temporarer, partieller Leberverlagerung. Fortschr Rontgenstr 16: 173207.

2. Moaven O, Hodin RA (2012) Chilaiditi Syndrome. Gastroenterol Hepatol (N Y) 8: 276-278. [Crossref]

3. Oldfield AL, Wilbur AC (1993) Retrogastric colon: CT demonstration of anatomic variations. Radiology 186: 557-561. [Crossref]

4. Poppel MH (1960) Duodenocolic apposition. Am J Roentgenol Radium Ther Nucl Med 83: 851-856. [Crossref]

5. Bredolo F, Esposito A, Casiraghi E, Cornalba G, Biondetti P (2011) Intestinal interposition: the prevalence and clinical relevance of non-hepatodiaphragmatic conditions (non-Chilaiditi forms) documented by CT and review of the literature. Radiol Med 116: 607-619. [Crossref]

6. Jose T, Ramachandran TM, Sebastian S, Thomas V (2007) Retrogastric interposition of colon: differential diagnosis for gastric pseudo-tumor. Indian J Gastroenterol 26: 141.

7. Nagayoshi K, Nakanishi K (2000) Retrogastric colon mimicking foramen of Winslow hernia or left paraduodenal hernia: case report. Nihon Igaku Hoshasen Gakkai Zasshi 60: 445-447. [Crossref]

8. Harpain F, Gasché C, Prager G, Silberhumer GR (2018) A siphon-like retrogastric transverse colon: A case report. Int J Surg Case Rep 44: 110-113. [Crossref]

9. Unal B, Kara S, Aktaş A, Bilgili Y (2004) Anatomic variations of the colon detected on abdominal CT scans. Tani Girisim Radyol 10: 304-308. [Crossref]

Copyright: (C2020 Stalin V. This is an open-access article distributed under the terms of the Creative Commons Attribution License, which permits unrestricted use, distribution, and reproduction in any medium, provided the original author and source are credited. 